\title{
A P-band 5-way Unequal Split High Power Divider for SAR Applications
}

\author{
Alberto Di Maria, Markus Limbach, Ralf Horn, Andreas Reigber \\ Microwaves and Radar Institute \\ German Aerospace Center (DLR) \\ Oberpfaffenhofen, Germany \\ Alberto.DiMaria@dlr.de
}

\begin{abstract}
The design and test of 5-way high power divider operating at P-band for the airborne Synthetic Aperture Radar (SAR) system of DLR is presented. Distinctive features are high bandwidth, high power and an unequal power split on the 5 output ports.
\end{abstract}

Keywords - P-band; Synthetic Aperture Radar; power divider; unequal split.

\section{INTRODUCTION}

The Microwaves and Radar Institute at the German Aerospace Center in Oberpfaffenhofen operates an airborne imaging radar named F-SAR [1]. It is a highly flexible Synthetic Aperture Radar (SAR) that is able to operate in a wide range of frequencies starting from $435 \mathrm{MHz}$ (P-band) up to $9.5 \mathrm{GHz}$ (X-band). With this radar system it is possible to test several polarimetric and interferometric techniques and their corresponding processing algorithms. It is also an excellent test bed for investigating the expected system performance when designing SAR satellites (Fig. 1).

In P-band (435 MHz) this radar is able to operate in dual linear polarization with $800 \mathrm{~W}$ pulse power $(10 \%$ duty cycle $)$ and $70 \mathrm{MHz}$ bandwidth. As the footprint on the ground of the radiated beam directly affects the SAR performance, the beamwidth, the side lobe level (SLL) and the stability of the beam shape over the frequency band are key specifications for

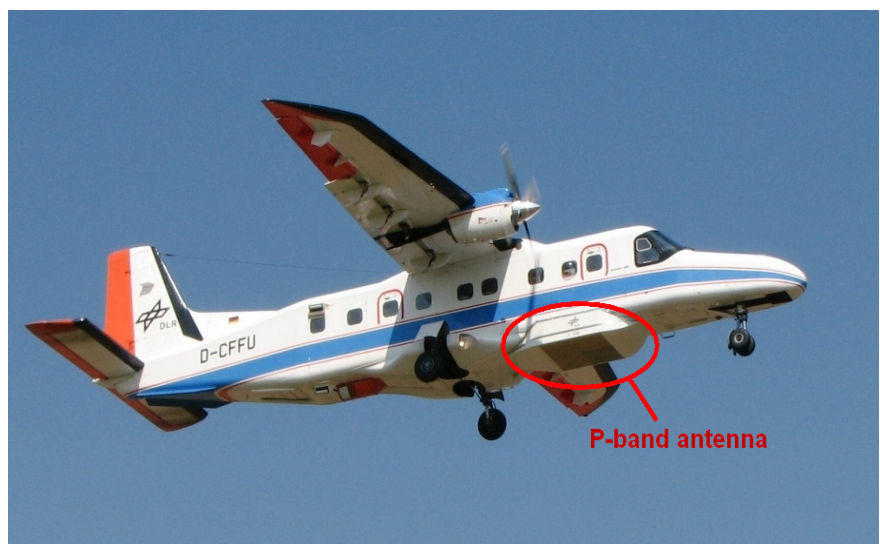

Fig. 1. The P-band antenna mounted under the aircraft Do-228. the antenna.

The developed P-band antenna is a $5 \times 5$ array mounted under the fuselage of the airplane and its radiating elements are patch-loaded cavity resonators. The antenna is dual linearly polarized and it has two operating configurations: nadirlooking and side-looking (Fig. 2). A corporate feeding network is used to give proper amplitude and phase to each element for each polarization and for each operational mode.

The amplitudes and the phases of the excitations required for nadir-looking mode are trivial: as the antenna beam is symmetric and directed downward, the array is fed with uniform phase (no steering) and with a symmetric tapered
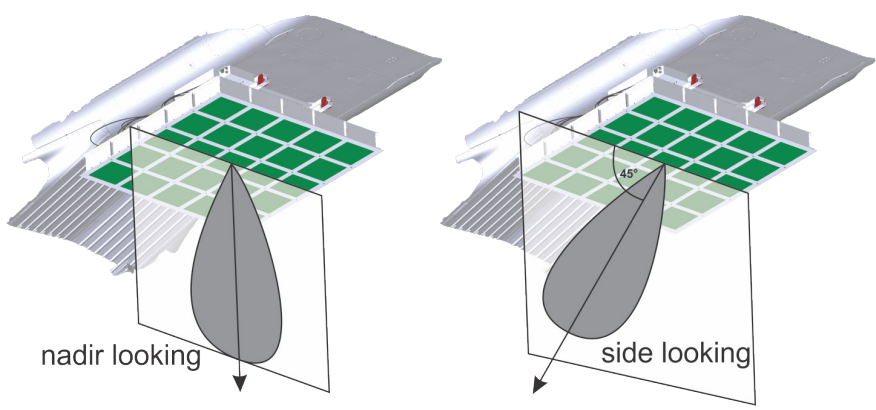

Fig. 2. Sketch of the beam on the elevation cut for the nadir-looking and the side-looking configurations.

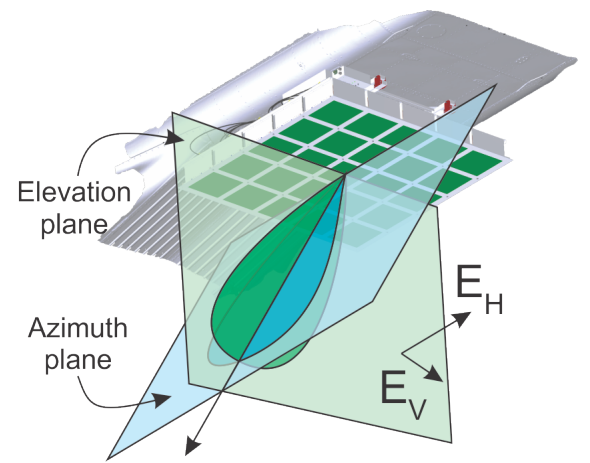

Fig. 3. Elevation and azimuth planes definition. The antenna polarization is defined horizontal (H-pol) when the radiated electric field is perpendicular to the elevation plane (like $\mathrm{E}_{\mathrm{H}}$ ); it is defined as vertical (V-pol) when the electric filed lies on the elevation plane $\left(\mathrm{E}_{\mathrm{V}}\right)$. 
amplitude to lower the side lobes (specifically a $\cos ^{2}$ tapering is used). Consequently, the design of a suitable power splitter doesn't present particular difficulties (with the exception of the high power handling capability, the bandwidth and an odd number of output ports [2]).

When the antenna is operating in side-looking mode, the beam requirements became tight. On the elevation plane the main beam should point at 45 degrees and the $3 \mathrm{~dB}$ beamwidth should be at least 35 degrees (elevation and azimuth planes are defined in Fig. 3). Then it is required to minimize as much as possible the signal received from all other directions in order to avoid ambiguities. That means that the gain in the opposite swath direction should be at least $20 \mathrm{~dB}$ lower than the maximum antenna gain. In addition, as the antenna is going to be mounted under the fuselage of the airplane, the radiation pattern should present a null in nadir direction to suppress the direct reflection of the ground. Finally, the radiation pattern for the $\mathrm{H}$ and $\mathrm{V}$ polarizations should be as equal as possible, at least in the main beam area (see again Fig. 3 for $\mathrm{H}$ and $\mathrm{V}$ definition). One may think that the latter should be automatically satisfied, but unfortunately this is not the case. In fact the radiating element is not symmetric respect to the polarization and each of them is rotated respect to the others in order to minimize the cross-pol level. In short, $\mathrm{H}$ and $\mathrm{V}$ polarizations require (slightly) different excitations in order to obtain the same radiation pattern.

This paper details the design of a power divider able to provide the necessary amplitude tapering in order to meet the radiation requirements in the elevation cut for the side-looking mode. Then, a realized prototype is shown and, finally, the measurement results are presented.

\section{OPTIMIZATION OF THE ARRAY ELEMENTS AMPLITUDE AND PHASE}

An array antenna is conventionally fed with a symmetric

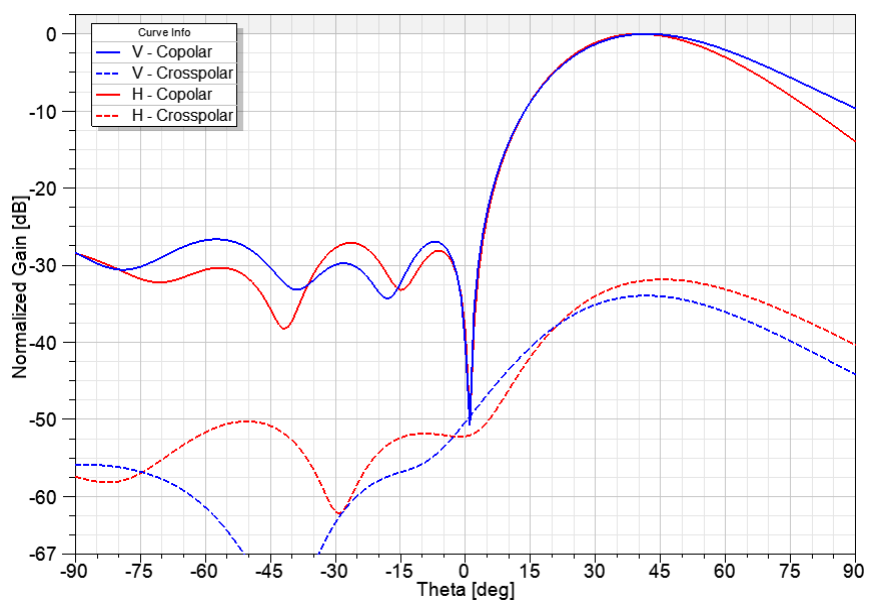

Fig. 4. Normalized antenna gain for the side looking configuration. Blue is $\mathrm{V}$-pol, red is H-pol, solid line is copolar and dashed line is cross-polar. For the $\mathrm{H}$ polarization the amplitude and phase values are $a=[0.0499$ $\left.\begin{array}{lllll}0.2148 & 0.4138 & 0.2734 & 0.0482\end{array}\right] \mathrm{W}$ and $d=\left[\begin{array}{llll}93 & 106 & 107 & 113\end{array}\right] \mathrm{deg}$. For the $\mathrm{V}$ polarization the optimized values are $a=\left[\begin{array}{lll}0.0700 & 0.2922 & 0.3951\end{array}\right.$ $0.2140 \quad 0.0288] W$ and $d=\left[\begin{array}{llll}111 & 107 & 107 & 107\end{array}\right] \mathrm{deg}$

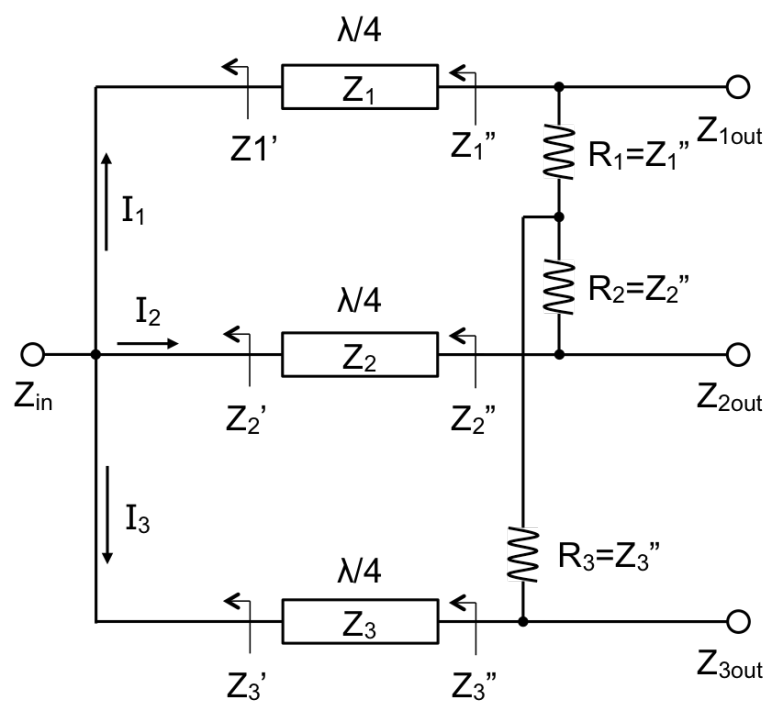

Fig. 5. Sketch of the 3-way unequal-split Wilkinson divider.

amplitude tapering and, when a beam steering is required, the relative phase of the excitations is changed by a constant value. In this application, given the amount of requirements and the reduced number of array elements in elevation direction, the amplitude and the phase for each element have been optimized independently. Let us call the amplitude values for the five elements $a_{1}, a_{2}, a_{3}, a_{4}$ and $a_{5}$ and their respective phases $p h_{1}$, $p h_{2}, p h_{3}, p h_{4}$ and $p h_{5}$.

The amplitudes are actually not independent. In fact, without losing in generality, the total input power can be considered unitary (and consequently each $a_{k}<1 \mathrm{~W}$ ). This can be expressed as

$$
a_{1}+a_{2}+a_{3}+a_{4}+a_{5}=1 W
$$

Then the following relation holds:

$$
a_{5}=1-\left(a_{1}+a_{2}+a_{3}+a_{4}\right)
$$

which shows that the amplitude value for one of the elements can be written as function of the others.

Even the phases are not fully independent: if the same amount of phase delay is added to all the five elements, there will be no effect on the radiation. Indeed, what really matters is the phase difference between two adjacent elements, defined as

$$
d_{k}=p h_{k+1}-p h_{k}
$$

with $k=1, \ldots, 4$ and being each $d_{k}=[0,180] \mathrm{deg}$.

The eight parameters have been optimized in order to obtain the desired radiation diagram. Fig. 4 shows the results for both polarizations at the end of the optimization process. As can be observed, all the requirements are met. 


\section{POWER DIVIDER DESIGN}

The required phases can be easily provided by adjusting the length of the cables connecting the array elements. The amplitude tapering must be generated instead by a power divider designed ad-hoc. The design process of the dividers for the two polarizations is identical and therefore only the one dedicated to the $\mathrm{H}$ polarization is presented in this paper.

As result of other design constraints, mainly due to flight certification requirements, the divider is realized in microstrip technology with a Rogers ${ }^{\circledR}$ substrate $1.27 \mathrm{~mm}$ thick and with $\varepsilon_{\mathrm{r}}=6.15$.

At the input stage a 3-way unequal-split Wilkinson is used. This design choice permits to solve the problem of the odd number of output ports: one arm is directly connected to the output, while the two others are further divided by 2-way splitters. These latter splitters are Lange couplers [5].

The design equations for the 3-way unequal-split Wilkinson can be derived as follows [3]

Referring to Fig. 5, the desired power division can be specified as the sum of the currents flowing from the input port down into each arm, and without losing generality, those currents can sum to $1 \mathrm{~A}$.

$$
\mathrm{I}_{1}+\mathrm{I}_{2}+\mathrm{I}_{3}=1
$$

In order to obtain the desired power split - or equivalently, current split -, each arm of the divider must present the appropriate impedance, which is called $Z_{k}{ }^{\prime}$ accordingly to Fig. 5. Given that the total current of $1 A$ is being delivered into a load of $Z_{\text {in }} \Omega$, the following relations must hold:

$$
Z_{1}{ }^{\prime}=Z_{\text {in }} / I_{1}, Z_{2}{ }^{\prime}=Z_{\text {in }} / I_{2}, Z_{3}{ }^{\prime}=Z_{\text {in }} / I_{3}
$$

For proper Wilkinson operation, there must be no current flowing in any of the isolation resistors when power is applied to the input port $\mathrm{P}_{\mathrm{in}}$. To obtain this, the voltage at the output side of each $\lambda / 4$ transmission line must be the same (let's say $V_{0}$ ) when power is applied to $\mathrm{P}_{\text {in }}$. As the voltage at the resistor section is equal to

$$
V_{k}=Z_{k} I_{k}
$$

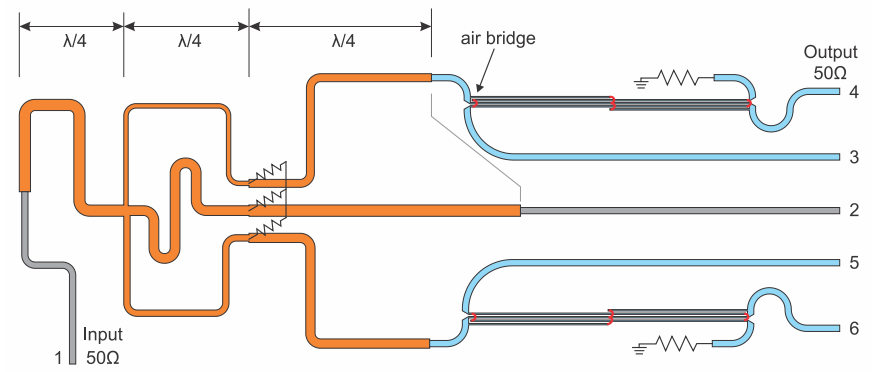

Fig. 6. The layout of the designed power divider. In orange the 3-way unequal-split Wilkinson and in light blue the Lange couplers.

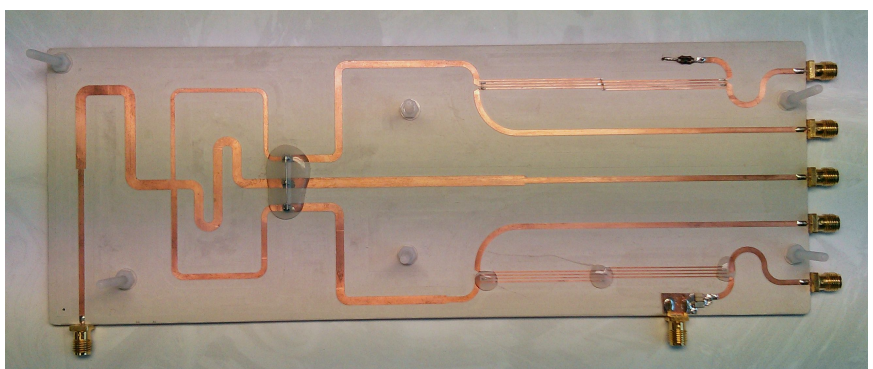

Fig. 7. Realized prototype. Two different kinds of terminations were tested.

the following must be satisfied:

$$
Z_{1} I_{1}=Z_{2} I_{2}=Z_{3} I_{3}=V_{0}
$$

It is worth noting that the previous holds even in case of an unequal split of the input power: it doesn't matter if a different amount of current flows down each arm, at this section the voltage on each arm is the same.

The impedance $Z_{k}{ }^{\prime \prime}(k=1,2,3)$, seen when looking into the output of each arm of the divider is equal to

$$
Z_{1}^{\prime \prime}=Z_{1}{ }^{2} / Z_{1}^{\prime}, Z_{2}^{\prime \prime}=Z_{2}{ }^{2} / Z_{2}^{\prime}, Z_{3}^{\prime \prime}=Z_{3}^{2} / Z_{3}^{\prime}
$$

as each $\lambda / 4$ transmission line is actually an impedance transformer from $Z_{k}$ ' to $Z_{k}$ "'.

Finally, as for the N-way equal split Wilkinson [4], the isolation resistor values for a star configuration divider are equal to the $Z_{k}$ " values for each arm of the divider.

With the aid of (1), (4) and (5) the design of the 3-way unequal-split Wilkinson located at the first stage has been carried out. Fig. 6 shows its layout colored in orange. In order to have reasonable line widths for the given impedance ratios, extra $\lambda / 4$ transformers are inserted at the input and the output ports as shown in Fig. 6.

The middle arm is designed to hold the higher power and it is directly connected to the output port. In this way there is an uninterrupted path between the input and the port with the higher output power (port 2): this solution increases the power handling and minimizes the losses.

The current flowing into the external arms is further split by Lange couplers. Fig. 6 shows their layout colored in light blue. This kind of interdigitated quadrature couplers solve the problem associated with two-line edge couplers in microstrip, which is that to achieve the correct capacitance between the strips the gaps become too small to manufacture. The Lange coupler is a bit more complicated to manufacture respect to the Waugh version of the coupler [6] (sometimes called four-line or unfolded Lange) as it has an air bridge also at the center, but it guaranties higher performances especially in terms of bandwidth. The coupler has been designed using the design equation provided by [7].

A further optimization of both couplers was necessary to get the best matching and the correct amplitude split. At the isolated port of the Lange couplers a small capacitance $(1 \mathrm{pF})$ 


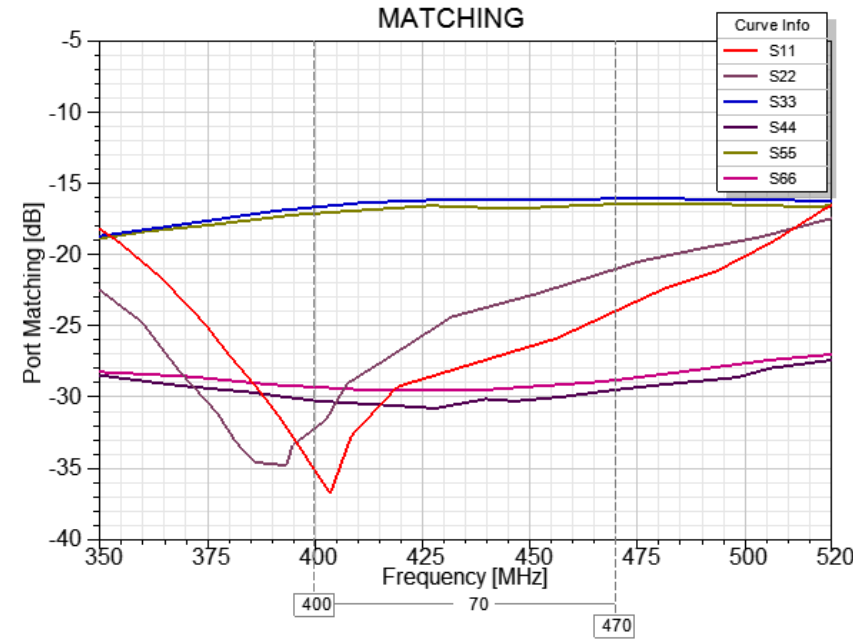

Fig. 8. Measurement results of the prototype: port matching. The ports are numbered according to Fig. 6 . The markers on the $\mathrm{x}$ axis highlight the desired bandwidth.

has been used in parallel to the $50 \Omega$ termination in order to compensate the small reactance seen at this port.

\section{MEASUREMENTS}

A prototype of the coupler was realized and measured with a R\&S® ZVA24 vector network analyzer. Fig. 7 shows a picture of the prototype. Two different kinds of terminations were tested; the main difference between them is the heat dissipation capability as can be seen in the high power measurements.

Fig. 8 and Fig. 9 show the measurements results. As can be observed the matching is very good and the transmission effectively realize the desired values and it is constant over the entire bandwidth.

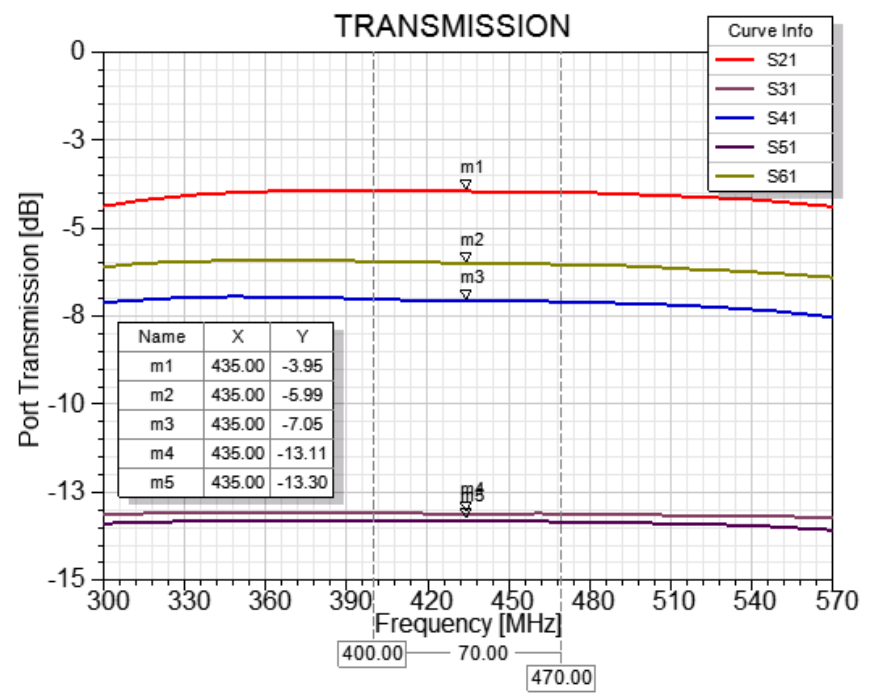

Fig. 9. Measurement results of the prototype: port transmission. The ports are numbered according to Fig. 6. The markers on the $\mathrm{x}$ axis highlight the desired bandwidth. The desired amplitude values in $\mathrm{dB}$ are $\mathrm{S}_{31}=-13.1, \mathrm{~S}_{41}=-6.81, \mathrm{~S}_{21}=-3.95, \mathrm{~S}_{61}=-5.75, \mathrm{~S}_{51}=-13.3$.

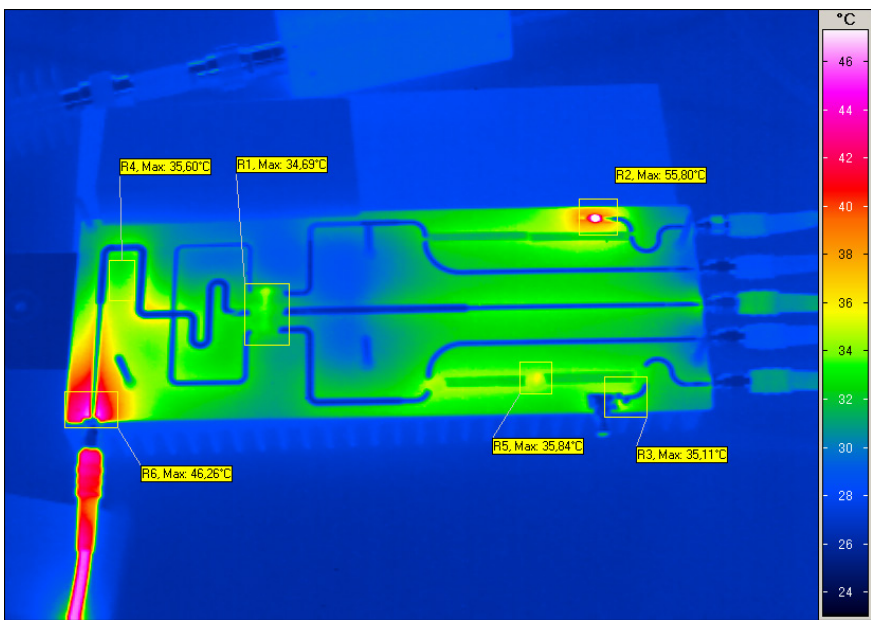

Fig. 10. Infrared picture of the divider under high power measurements. This picture was taken after 15 minutes at $60 \mathrm{dBm}$ with $10 \%$ duty cycle. Two different kinds of terminations were tested (marker R3 and R2) and it can be seen that they have a very different thermal performance.

The total insertion losses are $0.25 \mathrm{~dB}$ and this reflects positively in the high power measurements. The prototype has been tested up to a maximum of $60 \mathrm{dBm}(1 \mathrm{~kW})$ peak power with a duty cycle of $10 \%$. Fig. 10 shows an infrared picture of the divider under stress. The device can very well withstand to the maximal power.

\section{CONCLUSIONS}

A 5-way unequal-split power divider operating in P-band has been designed. The desired power division has been achieved by using a 3-way unequal-split Wilkinson in conjunction with two Lange couplers. A prototype has been built and measured and it shows very good performances.

\section{REFERENCES}

[1] R. Horn, A. Nottensteiner, A. Reigber, J. Fischer, R. Scheiber, "FSAR-DLR's new multifrequency polarimetric airborne SAR." Geoscience and Remote Sensing Symposium, 2009 IEEE International, IGARSS 2009. Vol. 2. IEEE, 2009.

[2] Kosc, A., Di Maria, A., Limbach, M.; Horn, R., Reigber, A., "A 5 way lumped-elements Wilkinson power divider," Antennas and Propagation (EuCAP), 2013 7th European Conference on, pp. 357, 360, 8-12 April 2013.

[3] "N-way Unequal-Split Wilkinsons", Microwave Encyclopedia Microwaves101.com,

http://www.microwaves101.com/encyclopedias/654-n-way-unequalsplit-wilkinsons, Retrieved on $1^{\text {st }}$ July 2014.

[4] Wilkinson, E.J., "An N-Way Hybrid Power Divider," Microwave Theory and Techniques, IRE Transactions on , vol.8, no.1, pp.116,118, January 1960

[5] Lange, J., "Interdigitated Stripline Quadrature Hybrid (Correspondence)," Microwave Theory and Techniques, IEEE Transactions on , vol.17, no.12, pp.1150,1151, Dec 1969

[6] Waugh, R.; Lacombe, D., "Unfolding the Lange Coupler (Short Papers)," Microwave Theory and Techniques, IEEE Transactions on , vol.20, no.11, pp.777,779, Nov. 1972

[7] Ou, W.P., "Design Equations for an Interdigitated Directional Coupler (Short Papers)," Microwave Theory and Techniques, IEEE Transactions on , vol.23, no.2, pp.253,255, Feb 1975 